\title{
Design and Simulation of Graphene/2D Interlayer Surface Plasmon Resonance Biosensor Based on Ellipsometry Method
}

\author{
Mohammad Javad Haji Najafia, Seyedeh Bita Saadatmand ${ }^{a}$, Vahid Ahmadia,"*, and Seyedeh \\ Mehri Hamidi ${ }^{b}$ \\ ${ }^{a}$ Optoelectronics and Nanophotonics Research Group, Faculty of Electrical and Computer \\ Engineering, Tarbiat Modares University, Tehran, Iran \\ ${ }^{\text {b}}$ Laser and Plasma Research Institute, Shahid Beheshti University, Tehran, Iran \\ “Corresponding author's Email: v_ahmadi@modares.ac.ir
}

\begin{abstract}
Regular paper: Received: Jan. 11, 2021, Revised: Mar. 7, 2021, Accepted: May. 6, 2021, Available Online: May. 8, 2021, DOI: 10.52547/ijop.15.1.27
\end{abstract}

\begin{abstract}
Two-dimensional nanomaterials have attracted increasing attention for enhancing surface plasmon resonance (SPR) biosensors application. In this work, we use the graphene layer to improve the sensitivity of the SPR biosensors based on the conventional Kretschmann configuration. We employ Tungsten disulfide $\left(\mathrm{WS}_{2}\right)$ and Molybdenum disulfide $\left(\mathrm{MoS}_{2}\right)$ Two-dimensional materials as an interlayer to enhance the sensitivity of $\mathrm{Au} / \mathrm{Graphene}$ biosensor in angle interrogation method. The transfer matrix method (TMM) is used to analyze the characteristics of the device. Results show that using $\mathrm{WS}_{2}$ in $\mathrm{Au} / \mathrm{Graphene}$ structure increases sensitivity by about $12.64 \%$, which is higher than $\mathrm{MoS}_{2}$. Combining graphene based SPR and ellipsometry as a highly sensitive, label-free, real-time, and versatile method can be used to measure a very small concentration of biomolecules, which leads to 170-fold enhancement compared to angle interrogation method and improves the detection accuracy and quality factor.
\end{abstract}

KEYWORDS: Ellipsometry, Graphene, Surface plasmon resonance, Transition metal dichalcogenides (TMD).

\section{INTRODUCTION}

Surface plasmon resonance (SPR) based biosensors have attracted great attention due to their high sensitivity, reliability, label-free, and ability for real-time detection [1]. Since the propagation constant of surface plasmon waves is larger than the light's propagation constant in vacuum, different methods are used to couple these two waves [2]. One of the most common methods is to use a prism with a refractive index higher than one, which was demonstrated by Kretschmann [3]. In this method, a transverse magnetic (TM)-polarized light passing through the prism at a certain angle and after total reflection, creates an evanescent wave at the interface between the metal and the prism. This evanescent wave penetrates the metal with the propagation constant $k_{x}=n_{p}(2 \pi / \lambda) \sin \theta$, where, $n_{p}, \lambda$, and $\theta$ are the refractive index of the prism, wavelength, and angle of the incident light, respectively [4]. The excitation of the surface plasmons results in a resonant dip in the angular/wavelength spectrum of the reflected light with a fixed excitation light angle/wavelength.

The most widely used metal in plasmonic sensors is gold $(\mathrm{Au})$ because it has a strong resonant response, chemical stability, and low loss [5]. Sensitivity enhancement and improving efficiency always are challenges in SPR based biosensors. Due to poor interaction between biomolecules with conventional Kretschmann SPR chip, the graphene layer was introduced as a biomolecular recognition element (BRE) [6]. Graphene, a twodimensional (2D)-material with a honeycomb structure, has a high aspect ratio, $\pi-\pi$ stacking interaction with biomolecules, and unusual optical properties [7]. So it provides a highly 
sensitive sensor and improves the efficiency of conventional prism/Au chip. Furthermore, due to effective charge transfer in $\mathrm{Au} / \mathrm{Graphene}$ interface, electric field enhancement is generated [8]. Physical adsorption of the biomolecules on the graphene surface leads to changes in the refractive index near the sensor surface and consequently induces specific alternations in the propagation constant and SPR characteristics [1], [4].

Also, 2D transition metal dichalcogenides (TMD) were proposed as interlayers to enhance the sensitivity of biosensors [9]. The large surface area and hydrophobic nature are special features of TMDs which makes them potential material to develop biosensing interfaces. Moreover, the TMD layers have been employed to inhibit the oxidation of metallic layers such as aluminum in SPR sensors. Y. Xu, et al. demonstrated that the Molybdenum disulfide $\left(\mathrm{MoS}_{2}\right)$-based SPR sensor possesses higher sensitivity and detection accuracy than graphene-based SPR sensors [10]. Due to the effective charge transfer, hybrid structures of $\mathrm{TMD} /$ Graphene lead to larger electric field enhancement at the sensing surface, so there will be higher sensitivity to analytes. However, the phase measurement of these structures has not been investigated in detail yet.

In this work, highly sensitive surface plasmon resonance enhanced ellipsometry, a novel method for sensitivity enhancement, has been used to probe the phase response of graphene based Kretschmann SPR structures. Then, the calculated sensitivity of these structures in the angle interrogation method has been compared to ellipsometry results.

\section{THEORY}

Figure 1 shows an SPR sensor that is based on the Kretschmann configuration. In the interlayer portion, Tungsten disulfide $\left(\mathrm{WS}_{2}\right)$ and $\mathrm{MoS}_{2}$ are used. The reflectance (R) of the structure shown in Fig. 2 can be calculated for TM polarized light with the N-Layer model [1], [2]. For TM polarized light, the reflectance is shown by $R$ as a function of matrix elements [6],

$$
R=\left|\frac{\left(M_{11}+M_{12} q_{N}\right) q_{1}-\left(M_{21}+M_{22} q_{N}\right)}{\left(M_{11}+M_{12} q_{N}\right) q_{1}+\left(M_{21}+M_{22} q_{N}\right)}\right|^{2},
$$

where

$$
M_{i j}=\left(\prod_{k=2}^{N-1} M_{k}\right)_{i j}, \quad i, j=1,2, \ldots,
$$

in which

$$
\begin{aligned}
& M_{k}=\left[\begin{array}{cc}
\cos \beta_{k} & -i \sin \beta_{k} / q_{k} \\
-i q_{k} \sin \beta_{k} & \cos \beta_{k}
\end{array}\right], \\
& \beta_{k}=d_{k}\left(\frac{2 \pi}{\lambda_{0}}\right)\left(\varepsilon_{k}-n_{1}^{2} \sin ^{2} \theta\right)^{1 / 2}, \\
& q_{k}=\frac{\left(\varepsilon_{k}-n_{1}^{2} \sin ^{2} \theta\right)^{1 / 2}}{\varepsilon_{k}},
\end{aligned}
$$

where, $\lambda_{0}$ is the wavelength of incident TMpolarized light, which is considered $633 \mathrm{~nm}, \theta$ is the incident angle, $n_{k}$ and $d_{k}$ are the refractive index (RI), and the thickness of the kth layer, respectively, with $k=2$ to $N-1$ [6]. The first layer is BK7 $(n=1.5151)$ prism or SF10 $(n=1.7231)$ prism. The $N$ th is the analyte defined as $n_{w}=1.33$ (water medium) and changes to $n_{b i o}=1.332$ as a biomolecule and immobilizer. The RI of $\mathrm{Au}$ is calculated with the Drude-Lorentz model, which is $0.1378+i 3.6196$ at $633 \mathrm{~nm}$. The thickness of monolayer graphene, $\mathrm{MoS}_{2}$, and $\mathrm{WS}_{2}$ is $0.34 \mathrm{~nm}, 0.65 \mathrm{~nm}$, and $0.8 \mathrm{~nm}$, respectively. Moreover, their corresponding refractive indices at $633 \mathrm{~nm}$ are $3+i 1.1487$, $5.0805+i 1.1723$, and $4.8937+i 0.3123$, respectively [6], [11]. The reflectance is related to change in RI of sensing medium, and dip in reflectance shows resonance angle (Fig. 2). As the RI of the sensing medium increases with $\Delta n$, the dip in reflectance shifts to a higher value that can be considered as $\Delta \theta$. To analyze sensor performance, sensitivity, detection accuracy (DA), and quality factor (QF) are the main parameters and should be as high as possible. Sensitivity is defined by $S=\frac{\Delta \theta}{\Delta n}[6]$. 
Detection accuracy is defined as the ratio of the shift in resonance angle $\left(\Delta \theta_{S P R}\right)$ to FWHM [12]:

$$
D A=\frac{\Delta \theta_{S P R}}{F W H M},
$$

In these biosensors, the quality factor is defined as the ratio of sensitivity to FWHM $\left(\mathrm{RIU}^{-1}\right)$ [12]:

$$
Q F=\frac{S}{F W H M},
$$

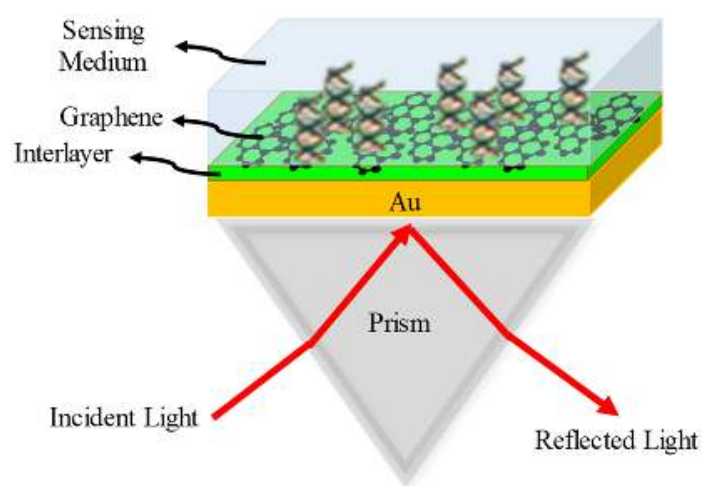

Fig. 1. The schematic of the proposed five-layer SPR structure.

Ellipsometry, a non-destructive and noncontact method, measures the polarization changes of a light passing through or reflected from a surface. In this technique, two highlysensitive quantities of $\Psi$ and $\Delta$ are calculated. $\Psi$ and $\Delta$ are the amplitude ratio and the phase difference between p-polarized and s-polarized light reflected from the sample surface, respectively. They are obtained as follows [13]:

$\tan \psi=\left|r_{p}\right| /\left|r_{S}\right| \rightarrow \psi=\tan ^{-1}\left[\frac{\sqrt{R_{p}}}{\sqrt{R_{S}}}\right]$,

where $r_{s, p}$ is the Fresnel reflection coefficient. Using the Kramers-Kronig relation, we have [13]:

$\operatorname{Ln}\left[r_{s, p}(\omega)\right]=\operatorname{Ln}\left[\sqrt{R_{s, p}^{(\omega)}}\right]+i \theta(\omega)$,

$$
\theta_{s, p}(\omega)=-\frac{2 \omega}{\pi} \Phi \int_{0}^{\infty} \frac{\operatorname{Ln}\left[{ }_{R \mathrm{~s}, \mathrm{p}}\left(\omega^{\prime}\right)\right.}{\omega^{\prime 2}-\omega^{2}} \mathrm{~d} \omega^{\prime}+\theta_{0},
$$

therefore, $\Delta$ is calculated as:

$$
\begin{aligned}
& \Delta=\theta_{p}(\omega)-\theta_{S}(\omega)= \\
& -\frac{2 \omega}{\pi} \Phi \int_{0}^{\infty}\left[\frac{\operatorname{Ln}\left[\sqrt{\frac{R_{p}\left(\omega^{\prime}\right)}{R_{S}\left(\omega^{\prime}\right)}}\right]}{\omega^{2}-\omega^{2}}\right] d \omega^{\prime}
\end{aligned}
$$

For ellipsometry measurement, we use the same approach proposed in [13].

\section{III.RESULTS AND DISCUSSION}

The graphene layer absorbs biomolecules because of its carbon rings. This property of graphene leads to larger refractive index changes at the interface between graphene and the sensing environment. Also, the presence of graphene on the surface of $\mathrm{Au}$ causes a change in surface plasmon propagation constant and thus increases the sensitivity to refractive index variation. The performance of SPR based biosensors is related to the sensitivity to refractive index changes and absorption efficiency of biomolecules on BRE which will be enhanced by the Graphene layer.

Figure 2(a) shows reflectance results for single layer graphene (SLG), bilayer graphene (Bi-G), trilayer graphene (Tri-G) and Fig. 2(b) shows $\mathrm{WS}_{2} / \mathrm{SLG}$ and $\mathrm{MoS}_{2} / \mathrm{SLG}$ structures in water (W) and biomolecule (Bio) medium on the BK7/Au substrate. Adding biomolecule to sensing medium leads to a slight change in the resonance angle of the SPR curve. This variation $\left(\Delta \theta_{\mathrm{SPR}}\right)$ in the dip of reflectance determines structure sensitivity, which means higher $\Delta \theta_{\mathrm{SPR}}$ leads to higher sensitivity. The results of the obtained curves shown in Figs 2(a) and 2(b) are listed in Tables 1 and 2, which give us information about different structures used in this work. From Table 1, $\mathrm{BK} 7 / \mathrm{Au} / \mathrm{WS}_{2} / \mathrm{SLG}$ has the best sensitivity in angle interrogation $\left(156^{\circ} / \mathrm{RIU}\right)$, better than 
$\mathrm{BK} 7 / \mathrm{Au} / \mathrm{MoS}_{2} / \mathrm{SLG}$ (152\%/RIU). Therefore, employing $\mathrm{MoS}_{2}$ and $\mathrm{WS}_{2}$ as an interlayer enhances the sensitivity, which has two main reasons. Firstly, the additional layer leads to an increase in the slope of $\theta-n_{d}$ dispersion, so by the change in RI of sensing medium, $\Delta \theta$ takes higher value as compared to the case of without interlayer. Secondly, effective charge transfers in $\mathrm{Au} / \mathrm{WS}_{2}$ or $\mathrm{MoS}_{2} /$ Graphene, lead to larger electric field enhancement, which results in higher sensitivity to sensing medium. In addition, the sensitivity in the cases with $\mathrm{WS}_{2}$ interlayers is enhanced more as compared with those with $\mathrm{MoS}_{2}$, due to the aforementioned reasons and its physical characteristics like refractive index.
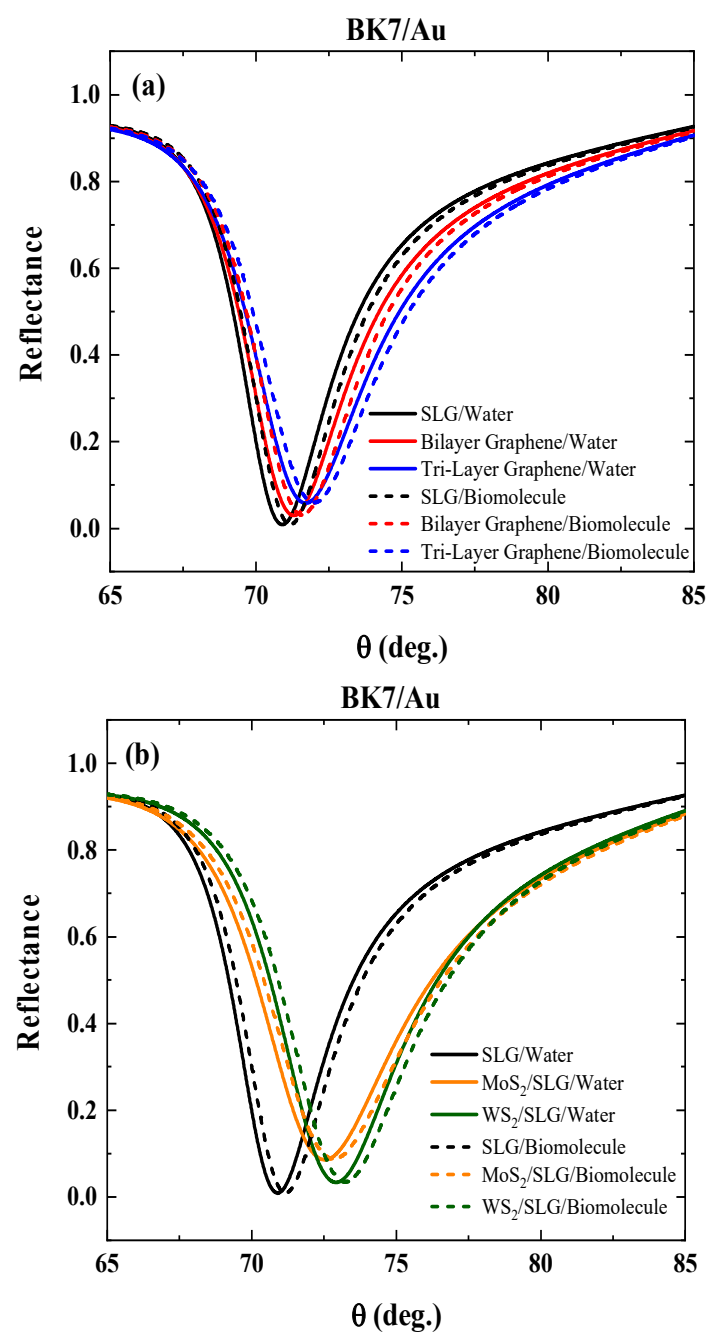

Fig. 2. SPR reflectance curves for (a) SLG, bilayer graphene, trilayer graphene, and (b) $\mathrm{WS}_{2} / \mathrm{SLG}$ and $\mathrm{MoS}_{2} / \mathrm{SLG}$ structures in water and biomolecules medium on the BK7/Au substrate.
Table 1. Simulation results for BK7/Au substrate, angle interrogation.

\begin{tabular}{|l|c|c|c|c|}
\hline \hline Structure & $\begin{array}{c}\theta_{\text {SPR }} \\
(\mathrm{W})\left(^{\circ}\right)\end{array}$ & $\begin{array}{c}\theta_{\text {SPR }} \\
\left(\mathrm{Bio}^{\circ}\left(^{\circ}\right)\right.\end{array}$ & $\begin{array}{c}\Delta \theta_{\text {SPR }} \\
\left({ }^{\circ}\right)\end{array}$ & $\begin{array}{c}\text { S } \\
(\% / R I U)\end{array}$ \\
\hline \hline SLG & 70.890 & 71.175 & 0.272 & 138.5 \\
\hline Bi-G & 71.288 & 71.571 & 0.283 & 141.5 \\
\hline Tri-G & 71.697 & 71.986 & 0.289 & 144.5 \\
\hline $\mathrm{WS}_{2} /$ SLG & 72.917 & 73.229 & 0.312 & 156 \\
\hline $\mathrm{MoS}_{2} / \mathrm{SLG}$ & 72.529 & 72.833 & 0.304 & 152 \\
\hline
\end{tabular}

Table 2. Simulation results for BK7/Au substrate, detection accuracy, and quality factor.

\begin{tabular}{|l|c|c|c|c|}
\hline \hline Structure & $\begin{array}{c}\text { FWHM } \\
(\mathrm{W})\left(^{\circ}\right)\end{array}$ & $\begin{array}{c}\text { FWHM } \\
(\mathrm{Bio})\left(^{\circ}\right)\end{array}$ & DA & $\begin{array}{c}\text { QF } \\
\left(\mathrm{RIU}^{-1}\right)\end{array}$ \\
\hline \hline SLG & 4.303 & 4.371 & 0.064 & 32.187 \\
\hline Bi-G & 4.826 & 4.874 & 0.059 & 29.320 \\
\hline Tri-G & 5.264 & 5.310 & 0.055 & 27.451 \\
\hline $\mathrm{WS}_{2} / \mathrm{SLG}$ & 5.450 & 5.478 & 0.057 & 28.620 \\
\hline $\mathrm{MoS}_{2} / \mathrm{SLG}$ & 5.778 & 5.799 & 0.053 & 26.307 \\
\hline
\end{tabular}

Table 2 shows a decline in detection accuracy and quality factor by employing an additional layer to SLG. This is due to additional optical absorption induced by adding graphene, $\mathrm{WS}_{2}$, and $\mathrm{MoS}_{2}$ layers.

Figure 3 shows the reflectance of TM and TE light, $\Psi$, and $\Delta$ for BK7/Au/SLG. As can be seen in Fig. 3, in the resonance angle, that is the dip in reflectance and $\Psi$, there is a linear and abrupt change in phase $(\Delta)$. This provides a very high sensitive measurement that can be used to monitor very slight changes in the sensing medium. Fig. 4 shows the phase response of different layers of graphene, $\mathrm{WS}_{2} / \mathrm{SLG}$, and $\mathrm{MoS}_{2} / \mathrm{SLG}$ in water and biomolecules medium on BK7/Au substrate. The results of Fig. 4 are listed in Table 3, which shows that phase response of all structures provides much higher sensitivity to measure a small change in sensing medium and $\mathrm{Au} / \mathrm{SLG}$ structure has the best sensitivity. In the $\mathrm{Au} / \mathrm{SLG}$ structure, the ellipsometry technique provides the phase sensitivity enhancement of more than 170 fold compared to angle interrogation. So, without adding any layer, high sensitive approach with a simple Au/Graphene SPR structure can be achieved by using the ellipsometry technique.

Using $\Psi$, we can define detection accuracy and quality factor in ellipsometry measurement. From Fig. 3, it appears that ellipsometry gives 
better DA and QF due to its narrower $\Psi$ spectrum. Results in Table 3 show that there is $24 \%$ to $60 \%$ increase in both detection accuracy and quality factor by using ellipsometry.

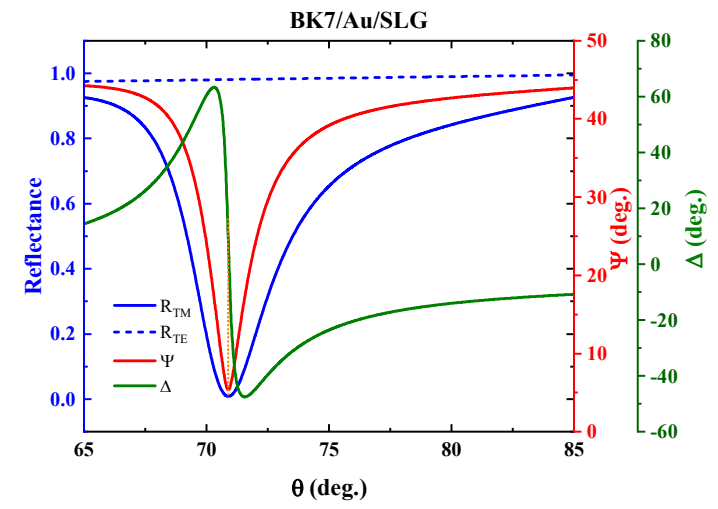

Fig. 3. Reflectance of TM (solid blue line) and TE (blue dashed line) light, $\Psi$ (red), and $\Delta$ (olive) for $\mathrm{BK} 7 / \mathrm{Au} / \mathrm{SLG}$. Orange vertical line shows that in resonance angle abrupt change in phase occurs.

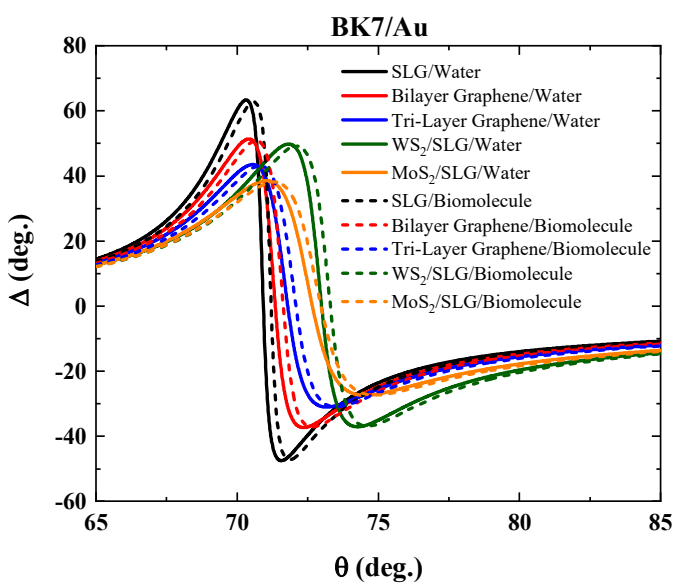

Fig. 4. Ellipsometry results for different structures.

So, by using ellipsometry not only much higher phase sensitivity is yielded but it also provides better resolution and quality factor, as a consequence overall performance of the SPR biosensor is improved.

Table 3. Ellipsometry results for $\mathrm{BK} 7 / \mathrm{Au}$ substrate, detection accuracy, and quality factor with $\Psi$ parameter

\begin{tabular}{|l|c|c|c|c|}
\hline \hline Structure & $\begin{array}{c}\Delta \\
\left({ }^{\circ}\right.\end{array}$ & $\begin{array}{c}\text { FWHM } \\
(\mathrm{W})\left(^{\circ}\right)\end{array}$ & DA & $\begin{array}{c}\text { QF } \\
\left(\mathrm{RIU}^{-1}\right)\end{array}$ \\
\hline SLG & 47.158 & 2.675 & 0.103 & 51.776 \\
\hline $\mathrm{Bi}-\mathrm{G}$ & 27.736 & 3.192 & 0.089 & 44.330 \\
\hline Tri-G & 17.835 & 3.765 & 0.077 & 38.380 \\
\hline $\mathrm{WS}_{2} / \mathrm{SLG}$ & 24.248 & 3.879 & 0.080 & 40.217 \\
\hline $\mathrm{MoS}_{2} / \mathrm{SLG}$ & 12.362 & 4.602 & 0.066 & 33.029 \\
\hline
\end{tabular}

Tables 4, 5, and 6 show the results of using SF10 prism instead of BK7, which has a lower sensitivity in angle interrogation. Ellipsometry results show that SF10 prism provides slightly lower phase sensitivity in graphene based structures. Although by adding TMD nanomaterials, SF10 based SPR sensors show better phase response compared to BK7 based SPR chip. This is due to a slightly sharper phase change which is caused by adding these layers. On the other hand, the detection accuracy and quality factor of BK7 based structures are better than SF10 based structures. In other words, by adding $\mathrm{WS}_{2}$ and $\mathrm{MoS}_{2}$, due to the physical characteristics of these materials, the slope of the $\Delta$ and the range of the phase changes decrease at the resonance angle for both BK7 and SF10 based structures. Consequently, the phase change by adding biomolecule decreases.

Table 4 Simulation results for SF10/Au substrate, angle interrogation

\begin{tabular}{|l|c|c|c|c|}
\hline \hline Structure & $\begin{array}{c}\theta_{\text {SPR }} \\
(\mathrm{W})\left(^{\circ}\right)\end{array}$ & $\begin{array}{c}\theta_{\text {SPR }} \\
\left(\mathrm{Bio}^{\circ}\left(^{\circ}\right)\right.\end{array}$ & $\begin{array}{c}\Delta \theta_{\text {SPR }} \\
\left({ }^{\circ}\right)\end{array}$ & $\begin{array}{c}\text { S } \\
\left({ }^{\circ} / \mathrm{RIU}\right)\end{array}$ \\
\hline \hline SLG & 59.397 & 56.545 & 0.148 & 74 \\
\hline Bi-G & 56.614 & 56.765 & 0.151 & 75.5 \\
\hline Tri-G & 56.840 & 56.993 & 0.153 & 76.5 \\
\hline $\mathrm{WS}_{2} /$ SLG & 57.501 & 57.659 & 0.158 & 79 \\
\hline $\mathrm{MoS}_{2} / \mathrm{SLG}$ & 57.303 & 57.459 & 0.156 & 78 \\
\hline
\end{tabular}

Table 5 Simulation results for SF10/Au substrate, detection accuracy, and quality factor.

\begin{tabular}{|l|c|c|c|c|}
\hline \hline Structure & $\begin{array}{c}\text { FWHM } \\
(\mathrm{W})\left(^{\circ}\right)\end{array}$ & $\begin{array}{c}\text { FWHM } \\
(\text { Bio })\left({ }^{\circ}\right)\end{array}$ & DA & $\begin{array}{c}\text { QF } \\
\left(\text { RIU }^{-1}\right)\end{array}$ \\
\hline \hline SLG & 2.269 & 2.282 & 0.065 & 32.613 \\
\hline Bi-G & 2.798 & 2.815 & 0.054 & 29.986 \\
\hline Tri-G & 3.091 & 3.109 & 0.050 & 24.749 \\
\hline $\mathrm{WS}_{2} /$ SLG & 3.230 & 3.248 & 0.049 & 24.458 \\
\hline $\mathrm{MoS}_{2} / \mathrm{SLG}$ & 3.535 & 3.554 & 0.044 & 22.065 \\
\hline
\end{tabular}

Table 6 Ellipsometry results for SF10/Au substrate, detection accuracy, and quality factor with $\Psi$ parameter

\begin{tabular}{|l|c|c|c|c|}
\hline \hline Structure & $\begin{array}{c}\Delta \\
\left({ }^{\circ}\right)\end{array}$ & $\begin{array}{c}\text { FWHM } \\
(\mathrm{W})\left(^{\circ}\right)\end{array}$ & DA & $\begin{array}{c}\text { QF } \\
\left(\mathrm{RIU}^{-1}\right)\end{array}$ \\
\hline \hline SLG & 47.105 & 1.564 & 0.095 & 47.310 \\
\hline Bi-G & 27.100 & 1.864 & 0.082 & 40.899 \\
\hline Tri-G & 17.804 & 2.153 & 0.071 & 35.532 \\
\hline $\mathrm{WS}_{2} / \mathrm{SLG}$ & 25.600 & 2.172 & 0.073 & 36.372 \\
\hline $\mathrm{MoS}_{2} / \mathrm{SLG}$ & 12.589 & 2.592 & 0.060 & 30.093 \\
\hline
\end{tabular}

However, this kind of decrease in the slope of the $\Delta$ is slightly lower in SF 10 based structures. Therefore, by adding TMD materials, the calculated phase change is slightly larger in 
SF10 based structures as compared to BK7 structures.

\section{IV.CONCLUSION}

Two dimensional transition metal dichalcogenides (TMD) materials like Tungsten disulfide $\left(\mathrm{WS}_{2}\right)$ and $\mathrm{MoS}_{2}$ have been used as an interlayer to enhance $\mathrm{Au} / \mathrm{Graphene}$ sensitivity. BK $7 / \mathrm{Au} / \mathrm{WS}_{2} / \mathrm{SLG}$ (single layer graphene) chip shows the most sensitivity in angle interrogation with an enhancement of about $12.64 \%$ compared to BK $7 / \mathrm{Au} / \mathrm{SLG}$. In angle interrogation, using BK7 prism leads to higher sensitivity compared to SF10 prism, but using TMD materials as an interlayer, SF10 based structures have shown better sensitivity in phase measurement. Ellipsometry results show $\mathrm{BK} 7 / \mathrm{Au} / \mathrm{SLG}$ has the best phase response. Using the ellipsometry technique as a non-destructive, high sensitive measurement not only provides phase sensitivity enhancement more than 170 fold compares to the angle interrogation, but it also improves the detection accuracy and quality factor of SPR biosensors.

\section{ACKNOWLEDGMENT}

This work was financially supported by Iran National Science Foundation (INSF). The authors would like to acknowledge the financial support received from Tarbiat Modares University, through grant \#IG-39703.

\section{REFERENCES}

[1] E. Wijaya, C. Lenaerts, S. Maricot, J. Hastanin, S. Habraken, J.-P. Vilcot, R. Boukherroub, and S. Szunerits "Surface plasmon resonance-based biosensors: From the development of different SPR structures to novel surface functionalization strategies," Current Opinion in Solid State and Materials Science, Vol. 15, pp. 208-224, 2011.

[2] M.S. Alexander, Plasmonics: Fundamentals and Applications, Springer Science \& Business Media, 2007.

[3] E. Kretschmann and H. Raether, "Radiative decay of non-radiative surface plasmons excited by light," Z. Naturforsch. A, Vol.23 pp. 2135-2136, 1968.
[4] H. Raether, Surface plasmons on smooth and rough surfaces and on gratings, Springer, Berlin, Heidelberg, 1988.

[5] E. Hutter and J.H. Fendler, "Exploitation of localized surface plasmon resonance," Adv. Mater. Vol.16, pp. 1685-1706, 2004.

[6] L. Wu, H.S. Chu, W.S. Koh, and E.P. Li, "Highly sensitive graphene biosensors based on surface plasmon resonance," Opt. Express, Vol.18, pp. 14395-14400, 2010.

[7] S. Szunerits, N. Maalouli, E. Wijaya, J.P. Vilcot, and R. Boukherroub, "Recent advances in the development of graphene-based surface plasmon resonance (SPR) interfaces," Anal. Bioanal. Chem, Vol. 405, pp. 1435-1443, 2013.

[8] S. Zeng, D. Baillargeat, H.-P. Ho, and K.-T. Yong, "Nanomaterials enhanced surface plasmon resonance for biological and chemical sensing applications," Chem. Soc. Rev. Vol. 43, pp. 3426-3452, 2014.

[9] M.S. Rahman, M.S. Anower, M.R. Hasan, M. B. Hossain, and M.I. Haque, "Design and numerical analysis of highly sensitive $\mathrm{Au}-$ $\mathrm{MoS}_{2}$-graphene based hybrid surface plasmon resonance biosensor," Opt. Commun. Vol. 396, pp. 36-43, 2017.

[10] YI. Xu, L. Wu, and L. Kee, "MoS highly sensitive near-infrared surface plasmon resonance refractive index sensor," IEEE J. Sel. Top. Quantum Electron. Vol. 25, pp. 1-7, 2018.

[11]C. Hsu, R. Frisenda, R. Schmidt, A. Arora, S. M. d. Vasconcellos, R. Bratschitsch, H.S.J.V.D. Zant, and A. Castellanos-Gomez "Thickness-dependent refractive index of $1 \mathrm{~L}$, 2L, and $3 \mathrm{~L} \mathrm{MoS}, \mathrm{MoSe}_{2}, \mathrm{WS}_{2}$, and $\mathrm{WSe}_{2}$," Adv. Opt. Mater. Vol. 7, pp. 1900239 (1-6), 2019.

[12] J. Maurya, Y. Prajapati, V. Singh, and J. Saini, "Sensitivity enhancement of surface plasmon resonance sensor based on graphene-MoS2 hybrid structure with $\mathrm{TiO} 2-\mathrm{SiO} 2$ composite layer," Appl. Phys. A, Vol.121, pp. 525-533, 2015.

[13] F. Sohrabi and S.M. Hamidi, "Optical detection of brain activity using plasmonic ellipsometry technique," Sens. Actuator B-Chem.Vol. 251, pp. 153-163, 2017. 


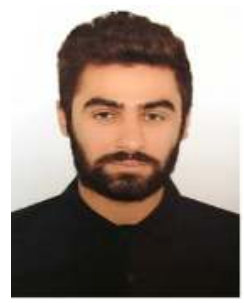

Mohammad Javad Haji Najafi received his B.Sc. degree in Electrical engineering from Shahid Beheshti University, Tehran, Iran (2017), M.Sc. degree in Electrical engineering from Tarbiat Modares University, Tehran, Iran (2020). His research interests include Biosensors, plasmonics, and nanophotonics.

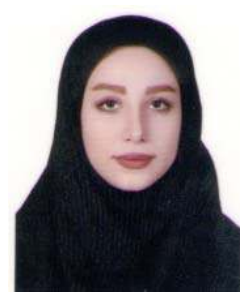

Seyedeh Bita Saadatmand received her B.Sc. degree in Electrical engineering from Shahid Beheshti University, Tehran, Iran (2016), M.Sc. degree in Electrical engineering from Tarbiat Modares University, Tehran, Iran (2020). She is currently a Ph.D. student in the Optoelectronic and Nanophotonic Lab., Faculty of Electrical and Computer Engineering, Tarbiat Modares University, Tehran, Iran. Her research interests include Biosensors, MEMS, plasmonics, and nanophotonics.

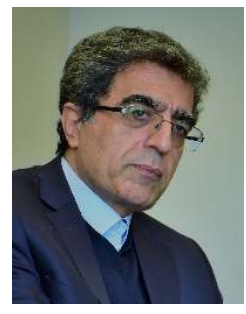

Vahid Ahmadi received his Ph.D. degree in Electrical Engineering from, Kyoto, Japan, in
1994. He is a Professor at TMU, Tehran, Iran. His current research interests include nanophotonics, plasmonics, and biophotonics, nonlinear optics, metamaterial-based devices, organic-based light sources and sensors, Perovskite-based solar cells, graphene, and 2D materials-based photonic devices. $\mathrm{He}$ is a member of the Founders-Board of Optics and Photonics Society of Iran; the editorial board of the International Journal of Information and Communication Technology Research, and Optical and Quantum Electronics (OQEL). He is the author/co-author of more than 380 scientific papers presented in journals and conferences. He is currently the chair of IEEE Iran-section and the Chair of the Optoelectronics and Photonics Research Group and the Head Nano-Optoelectronics Laboratory, TMU. He has also been the Editorin-Chief of Modares Journal of Electrical Engineering.

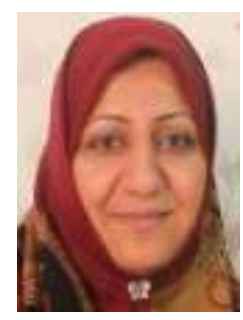

Seyedeh Mehri Hamidi received her Ph.D. degree in photonics from Laser and Plasma Research Institute, Shahid Beheshti University, Tehran, Iran, in 2009. She is currently the director of Magneto-Plasmonic Lab. of Laser and Plasma Research Institute. She works in the research fields of magneto-plasmonic, nanophotonics, neurophotonics, piezotonics, photonic and magnetophotonic crystals, Surface Plasmon Resonance, dielectric and magnetic nanostructures. 
THIS PAGE IS INTENTIONALLY LEFT BLANK. 